\title{
Roles of Aminoacyl-tRNA Synthetases in Cancer
}

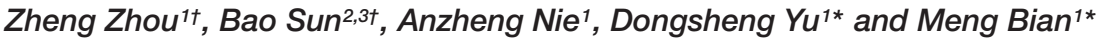 \\ ${ }^{1}$ Department of Chinese Medicine, The First Affiliated Hospital of Zhengzhou University, Zhengzhou, China, ${ }^{2}$ Department \\ of Pharmacy, The Second Xiangya Hospital, Central South University, Changsha, China, ${ }^{3}$ Institution of Clinical Pharmacy, \\ Central South University, Changsha, China
}

\section{OPEN ACCESS}

Edited by:

Massimiliano Berretta,

Aviano Oncology Reference Center

(IRCCS), Italy

Reviewed by:

Eduardo J. Salustiano,

Federal University of Rio de Janeiro,

Brazil

Zhichao $X$

Shanghai University of Traditional Chinese Medicine, China

*Correspondence: Dongsheng Yu

dongshyu@163.com Meng Bian

bianmeng0208@163.com

${ }^{\dagger}$ These authors have contributed equally to this work

Specialty section:

This article was submitted to Molecular and Cellular Oncology, a section of the journal Frontiers in Cell and Developmental Biology

Received: 28 August 2020 Accepted: 09 November 2020 Published: 27 November 2020

Citation:

Zhou Z, Sun B, Nie A, Yu D and Bian M (2020) Roles of Aminoacyl-tRNA Synthetases in Cancer. Front. Cell Dev. Biol. 8:599765. doi: $10.3389 /$ fcell.2020.599765
Aminoacyl-tRNA synthetases (ARSs) catalyze the ligation of amino acids to their cognate transfer RNAs (tRNAs), thus playing an important role in protein synthesis. In eukaryotic cells, these enzymes exist in free form or in the form of multi-tRNA synthetase complex (MSC). The latter contains nine cytoplasmic ARSs and three ARSinteracting multifunctional proteins (AIMPs). Normally, ARSs and AIMPs are regarded as housekeeping molecules without additional functions. However, a growing number of studies indicate that ARSs are involved in a variety of physiological and pathological processes, especially tumorigenesis. Here, we introduce the roles of ARSs and AIMPs in certain cancers, such as colon cancer, lung cancer, breast cancer, gastric cancer and pancreatic cancer. Furthermore, we particularly focus on their potential clinical applications in cancer, aiming at providing new insights into the pathogenesis and treatment of cancer.

\footnotetext{
Keywords: aminoacyl-tRNA synthetase, ARS-interacting multifunctional protein, cancer, pathogenesis, therapeutics
}

\section{INTRODUCTION}

The genetic information in the organism is transformed into functional proteins through transcription and translation. Of these, aminoacyl-tRNA synthetases (ARSs) catalyze the aminoacylation of transfer RNAs (tRNAs), thereby playing an important role in translation (Kwon et al., 2019). In this process, each ARS interacts with an amino acid and an ATP molecule to produce a high-energy aminoacyl adenylate (aa-AMP) intermediate and a pyrophosphate (PPi) molecule (Kwon et al., 2019). Subsequently, the intermediate binds to the cognate tRNA and transfers the amino acid to it. Eventually, the aminoacylated tRNA is recruited to the ribosome to participate in protein synthesis. Actually, there are thirty-six ARSs in a typical human cell, and they can function in the cytoplasm or mitochondria (Antonellis and Green, 2008). Of note, in addition to being in free form in the cytoplasm, nine ARSs interact with three ARS-interacting multifunctional proteins (AIMPs) to form a multi-tRNA synthetase complex (MSC) (Hyeon et al., 2019). Among them, AIMPs mainly play scaffolding roles in the assembly of the MSC. Furthermore, ARSs are usually divided into two categories based on their structural characteristics (Pang et al., 2014). The first is characterized by a Rossman fold in its catalytic domain, whereas the second has three conserved sequence motifs, namely Motif 1, Motif 2 and Motif 3 (Pang et al., 2014).

Generally, ARSs and AIMPs are considered as housekeeping molecules. However, increasing evidence indicates that these molecules are involved in various physiological and pathological processes, including cysteine polysulfidation, angiogenesis, posttranslational modification, immune 
response and nervous system development (Akaike et al., 2017; Fu et al., 2017; He et al., 2018; Mullen et al., 2020; Zhu et al., 2020). Strikingly, ARSs and AIMPs are closely related to tumorigenesis (Yin et al., 2016; Gao et al., 2019; Zhou Z. et al., 2020). Certain ARSs, such as isoleucyl-tRNA synthetase 2 (IARS2), lysyl-tRNA synthetase (KRS) and asparaginyl-tRNA synthetase (NRS), have been shown to promote cancer development (Kim et al., 2012; Fang et al., 2018; Yeom et al., 2020), while AIMPs and seryltRNA synthetase (SerRS) usually exert anti-tumor effects (Li Y. et al., 2019; Zhou Z. et al., 2020). Furthermore, some ARSs are abnormally expressed in various cancer tissues and have diagnostic and prognostic value (Jung et al., 2017; Lee and Kim, 2019). For instance, methionyl-tRNA synthetase (MRS) was highly expressed in non-small cell lung cancer (NSCLC) cells that had metastasized to lymph nodes but was weakly expressed in cells adjacent to the lesion (Lee and Kim, 2019). Importantly, MRS/CD45 dual immunofluorescent staining increased the diagnostic rate of lymph node metastasis in NSCLC, indicating that MRS might serve as a potential biomarker for cancer metastasis (Lee and Kim, 2019). In this review, we not only focus on the roles of ARSs and AIMPs in the development of cancer, but also emphasize the value of these molecules in the diagnosis and treatment of cancer.

\section{ROLES OF ARSS IN CANCER}

Besides the catalytic domains, ARSs also contain other domains, including leucine zipper, glutathione $S$-transferase, WHEP and endothelial monocyte activating polypeptide II domains, which seem to provide the possibility for the functional diversity of ARSs (Kwon et al., 2019). In fact, their abnormal expression, cellular localization and molecular interactions cause a variety of human diseases (Park et al., 2008; D’Hulst et al., 2020; Wu et al., 2020). Among them, some ARS-mediated signaling pathways are involved in the regulation of multiple tumors (Figure 1). Laminin activated p38/mitogen-activated protein kinase (MAPK) by binding to integrin, which induced the phosphorylation of KRS at the T52 residue in A549 cells (Kim et al., 2012). Subsequently, the phosphorylated KRS dissociated from the MSC and interacted with $67-\mathrm{kDa}$ laminin receptor (67LR) that was localized in the cell membrane. This interaction prevented neural precursor cell expressed developmentally downregulated 4 (NEDD4) from ubiquitinating 67LR as well as enhanced laminin-induced cell migration (Kim et al., 2012). Analogously, Nam et al. found that KRS played a vital role in the invasive dissemination of colon cancer spheroids in 3D collagen I gels (Nam et al., 2015). Membranous KRS formed a complex with 67LR and integrin $\alpha 6 \beta 1$ to mediate extracellular signal-regulated kinase $1 / 2(\mathrm{ERK} 1 / 2) / \mathrm{c}-J u n$ activation and paxillin expression in colon cancer cells, thereby promoting cancer dissemination (Nam et al., 2015). These findings indicate that KRS plays an important role in cancer metastasis.

Moreover, certain ARSs and AIMPs could regulate tumorigenesis by p53 (Park et al., 2005; Choi et al., 2011; Wang et al., 2018). High-fat diet induced colonic lysine homocysteinylation through MRS, which led to the accumulation of DNA damage and colorectal cancer-like phenotypes in the colon of mice and rats (Wang et al., 2018). Specifically, MRS increased the homocysteinylation of ataxia telangiectasia and Rad3-related protein (ATR) at five lysine residues by binding to ATR, thereby inhibiting ATR and its downstream moleculars checkpoint kinase-1 (CHK1) and p53 (Wang et al., 2018). Conversely, MRS inhibitors, $N$-acetyl cysteine and acetylhomocysteine thioether, could reverse tumor-like phenotypes in both HCT116 cells and rats, which provided new ideas for understanding the mechanism of high-fat dietassociated colorectal cancer (Wang et al., 2018). Considering that amino acids are the substrates of ARSs, high-protein diets or ketogenic diets may participate in tumor development by regulating ARSs, which deserves more in-depth research. Interestingly, a splicing variant of AIMP2 lacking exon 2 (AIMP2-DX2) was highly expressed in human lung cancer tissues, and the ratio of AIMP2-DX2 to AIMP2 was negatively correlated with patient survival (Choi et al., 2011). Further studies found that this splicing variant reduced the pro-apoptotic activity of AIMP2 by competitively binding to p53 with AIMP2 (Choi et al., 2011). Of note, AIMP3 heterozygous mice exhibited spontaneous tumorigenesis in multiple organs (Park et al., 2005). AIMP3 directly interacted with ataxia telangiectasiamutated (ATM)/ATR to up-regulate the expression of p53, thereby responding to DNA damage (Park et al., 2005). Under UV stimulation, the Ser662 site of MRS was phosphorylated by general control non-repressed-2 (GCN2), resulting in a conformational change of MRS and subsequent dissociation of AIMP3 from the MSC (Kwon et al., 2011). The released AIMP3 was translocated into the nucleus and participated in DNA repair (Kwon et al., 2011). Additionally, ARSs also contribute to the development of specific tumors via other pathways, such as colon cancer, lung cancer, breast cancer and pancreatic cancer (Shin et al., 2008; Katsyv et al., 2016; Jeong et al., 2018; Nam et al., 2018).

\section{ARSs and Colon Cancer}

Colon cancer is one of the most common gastrointestinal cancers and has the second and third highest mortality rates among male and female patients, respectively (Bin et al., 2020). Interestingly, KRS was involved in colon cancer metastasis by inducing M2 macrophage polarization (Nam et al., 2018). In this process, KRS dissociated from the MSC after S207 phosphorylation and then translocated to the nucleus (Nam et al., 2018). The nuclear KRS induced growth arrest-specific 6 (GAS6) transcription by activating microphthalmia-associated transcription factor (MiTF), which promoted the M2 polarization of neighboring macrophages (Nam et al., 2018). Subsequently, M2 macrophages secreted soluble factors, such as growth-regulated oncogene- $\alpha$ (GRO $\alpha$ ), fibroblast growth factor 2 (FGF2) and macrophage colony-stimulating factor (M-CSF). These factors not only induced the activation of intracellular signals in cancer cells, but also activated the adjacent cancer-associated fibroblasts and promoted the secretion of laminins, which ultimately remodeled the tumor microenvironment and exacerbated cancer metastasis (Nam et al., 2018). Furthermore, caspase-8 could cleave KRS and expose the PDZ binding motif located at the C-terminus of KRS 


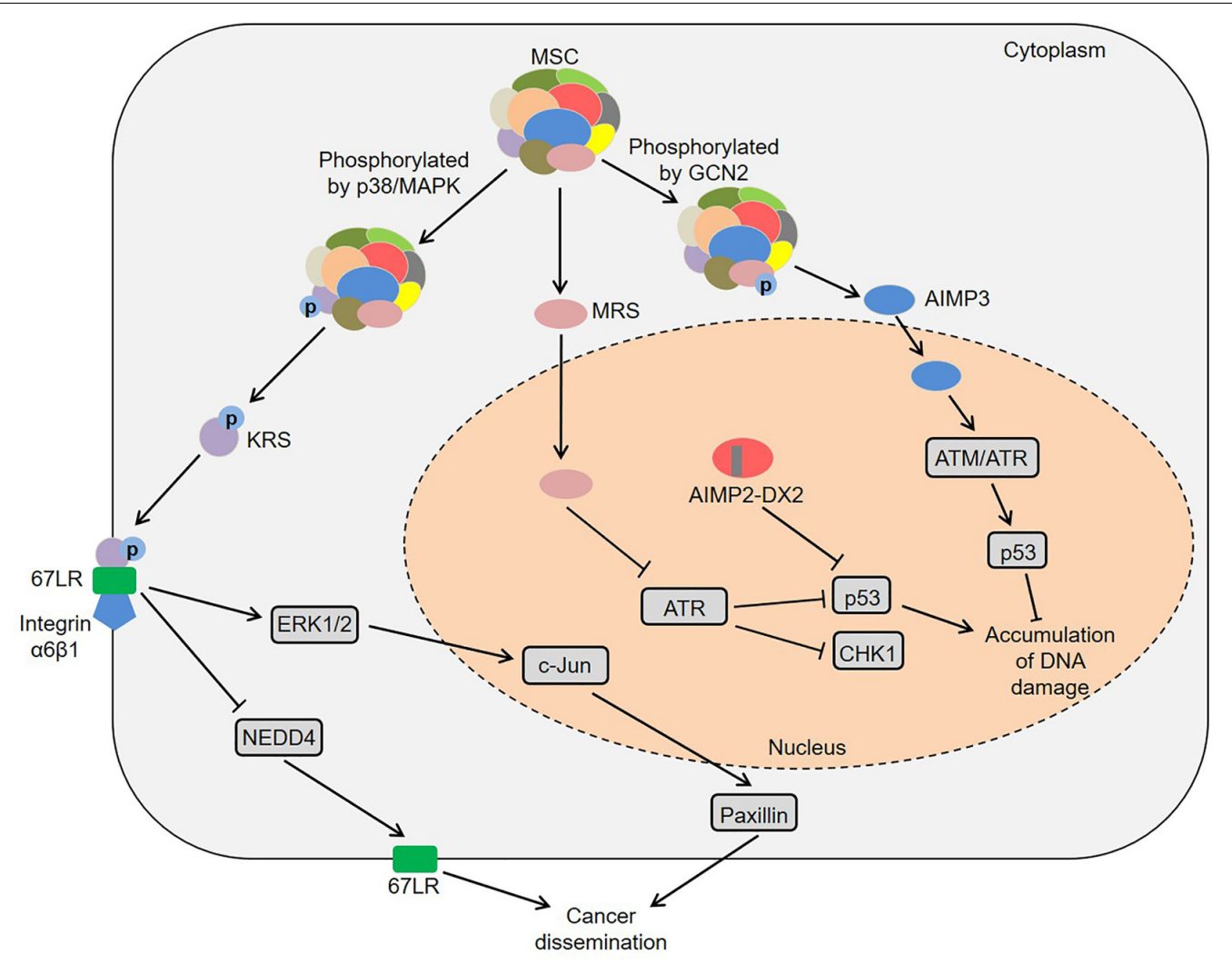

FIGURE 1 | Common pathways mediated by ARSs in cancer development. KRS is phosphorylated by p38/MAPK and then dissociates from the MSC. Subsequently, the KRS interacts with 67LR that is localized in the cell membrane, which prevents NEDD4 from ubiquitinating 67LR and enhances laminin-induced cell migration. Moreover, KRS forms a complex with 67LR and integrin $\alpha 6 \beta 1$ to mediate ERKs/c-Jun activation and paxillin expression. MRS increases the lysine homocysteinylation of ATR, thereby inhibiting ATR and its downstream CHK1 and p53. AIMP2-DX2 reduces the pro-apoptotic activity of AIMP2 by competitively binding to $\mathrm{p} 53$ with AIMP2. Furthermore, MRS is phosphorylated by GCN2, resulting in a conformational change of MRS and subsequent dissociation of AIMP3 from the MSC. The released AIMP3 interacts with ATM/ATR to up-regulate the expression of p53, thereby responding to DNA damage. ARSs, aminoacyl-tRNA synthetases; AIMPs, ARS-interacting multifunctional proteins; KRS, lysyl-tRNA synthetase; MAPK, mitogen-activated protein kinase; MSC, multi-tRNA synthetase complex; 67LR, 67-kDa laminin receptor; NEDD4, neural precursor cell expressed developmentally downregulated 4; ERKs, extracellular signal-regulated kinases; MRS, methionyl-tRNA synthetase; ATR, ataxia telangiectasia and Rad3-related protein; CHK1, checkpoint kinase-1; AIMP2-DX2, AIMP2 lacking exon 2; GCN2, general control non-repressed-2; ATM, ataxia telangiectasia-mutated.

in HCT116 cells (Kim S.B. et al., 2017). The exposed PDZ binding motif interacted with syntenin, facilitating the dissociation of KRS from the MSC and the subsequent exosomic secretion of KRS from colorectal carcinoma cells. Importantly, the KRScontaining exosomes induced the M1 polarization and migration of macrophages, indicating that KRS might be involved in cancerinduced inflammation (Kim S.B. et al., 2017). These results suggest that KRS is closely associated with the development of colon cancer.

Zhong et al. (2015) observed that IARS2 was highly expressed in human colon cancer tissues, and knockdown of IARS2 could inhibit RKO cell proliferation, suggesting that IARS2 might trigger the development of colon cancer. Furthermore, NRS induced Yorkie-mediated tumor phenotypes in a Drosophila model (Yeom et al., 2020). During this process, NRS blocked the interaction between Salvador and Hippo by binding to Salvador, thereby activating Yorkie target genes via decreasing Yorkie phosphorylation (Yeom et al., 2020). Notably, YAP, a mammalian homolog of Yorkie, target genes were upregulated in colon cancer C26 cells, and NRS inhibitor TirB decreased the levels of YAP target genes and suppressed cell proliferation in C26 cells, indicating that NRS might regulate the development of colon cancer by Hippo signaling pathway (Yeom et al., 2020). Intriguingly, the hemizygous deletion of AIMP2 promoted epithelial cell proliferation and intestinal stem cell expansion in the intestinal crypt, which resulted in the formation of intestinal adenoma in $A p c^{\mathrm{Min} /+}$ mice (Yum et al., 2016). Mechanistically, AIMP2 bound to Disheveled-1 (DVL1) and blocked the interaction between DVL1 and axis inhibition protein (AXIN), thus inhibiting the activity of Wnt/ $\beta$-catenin signaling (Yum et al., 2016). In short, ARSs and AIMPs are closely related to the biology of colon cancer (Figure 2).

\section{ARSs and Lung Cancer}

Lung cancer is the leading cause of cancer-related deaths worldwide, with dismal prognosis and poor clinical outcomes (Drula et al., 2020). Previous studies found that leucyl-tRNA synthetase (LRS) was significantly up-regulated in A549 lung 


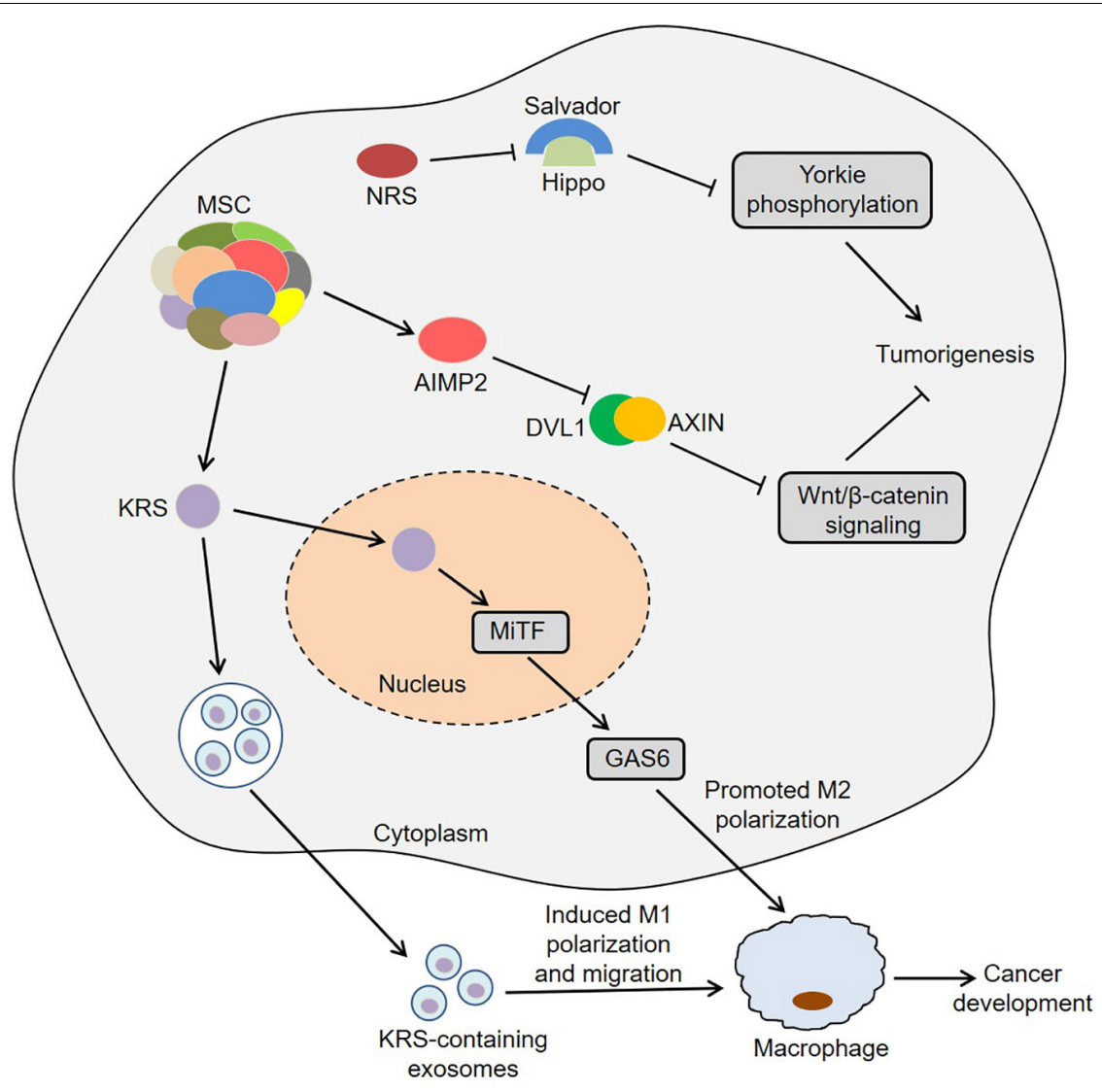

FIGURE 2 | Roles of ARSs and AIMPs in the biology of colon cancer. KRS translocates to the nucleus and then induces GAS6 transcription by activating MiTF, which promotes the M2 polarization of neighboring macrophages and then facilitates cancer metastasis. KRS-containing exosomes are released by cancer cells and then induce M1 polarization and migration of macrophages. NRS blocks the interaction between Salvador and Hippo by binding to Salvador, thereby activating Yorkie target genes via decreasing Yorkie phosphorylation. AIMP2 blocks the interaction between DVL1 and AXIN and thus inhibits the activity of Wnt/ $\beta$-catenin signaling. ARSs, aminoacyl-tRNA synthetases; AIMPs, ARS-interacting multifunctional proteins; KRS, lysyl-tRNA synthetase; MiTF, microphthalmia-associated transcription factor; GAS6, growth arrest-specific 6; NRS, asparaginyl-tRNA synthetase; DVL1, disheveled-1; AXIN, axis inhibition protein.

cancer cells, and its mRNA was also highly expressed in primary lung cancer tissues (Shin et al., 2008). Importantly, the growth and migration of LRS knockdown A549 cells were inhibited, indicating that this molecule played an important role in the development of lung cancer (Shin et al., 2008). Furthermore, tyrosyl-tRNA synthetase (TyrRS) and microtubuleactin crosslinking factor 1 (MACF-1) were at higher abundance in lung adenocarcinoma tissues than adjacent normal tissues (Zhou et al., 2013). Among them, the levels of TyrRS were difference between N1, N2, and N3, while the levels of MACF-1 were associated with $\mathrm{T}$ stage, $\mathrm{N}$ stage and clinical stage. Meanwhile, patients expressing TyrRS or MACF-1 had a significantly increased risk of death (Zhou et al., 2013). Di et al. (2019) observed that the expression of IARS2 was higher in NSCLC tissues, and silencing IARS2 could inhibit the activity of A549 and H1299 cells and reduce the tumorigenicity of lung cancer cells in nude mice. Mechanistically, the phosphorylation levels of protein kinase $\mathrm{B}(\mathrm{AKT})$ and mammalian target of rapamycin (mTOR) were significantly lower in IARS2-silenced lung cancer cells, while AKT activator SC79 partially restored the AKT phosphorylation and lung cancer cell proliferation, suggesting that IARS2 might be involve in tumorigenesis through the AKT/mTOR pathway (Di et al., 2019).

In addition, AIMP2 and its splicing variant are involved in the development of lung cancer (Figure 3). AIMP2 had multidirectional tumor-suppressive activity, which mediated the apoptotic response to DNA damage, tumor necrosis factor $\alpha$ (TNF- $\alpha)$-induced cell death and sensitivity to antiproliferative transforming growth factor- $\beta$ (TGF- $\beta$ ) signal in a dose-dependent manner (Choi et al., 2009). Of note, heterozygous AIMP2 mice with low AIMP2 levels showed high susceptibility to lung and colon carcinogenesis (Choi et al., 2009). Kim et al. (2016) found that AIMP2 was phosphorylated by TGF- $\beta$-activated p38/MAPK at S156 in HeLa cells. Next, the phosphorylated AIMP2 dissociated from the MSC and translocated to the nucleus. The nuclear AIMP2 interacted with Smad ubiquitin regulatory factors 2 (Smurf2), thereby enhancing FUSE-binding protein (FBP) ubiquitination and down-regulating c-Myc (Kim et al., 2016). Meanwhile, the interaction between AIMP2 and Smurf2 also inhibited the binding of Smurf2 to chromosomal region maintenance 1 (CRM1), which enhanced TGF- $\beta$ signal by reducing the 


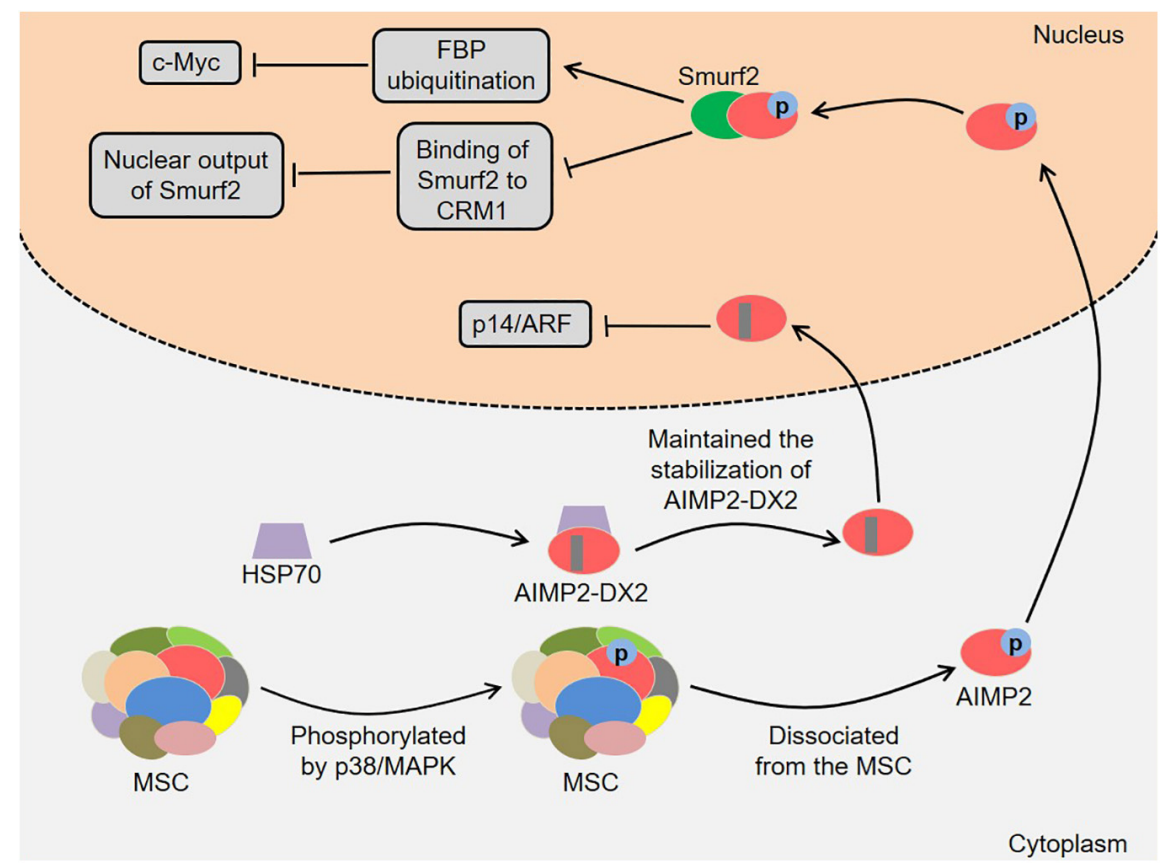

FIGURE 3 | Roles of AIMP2 and AIMP2-DX2 in the development of lung cancer. AIMP2 dissociates from the MSC and translocates to the nucleus. The nuclear AIMP2 interacts with Smurf2, thereby enhancing FBP ubiquitination and down-regulating c-Myc. Meanwhile, the interaction also inhibits the binding of Smurf2 to CRM1, which enhances TGF- $\beta$ signal by reducing the nuclear output of Smurf2. HSP70 inhibits the Siah1-dependent AIMP2-DX2 ubiquitination by interacting with AIMP2-DX2, thereby maintaining the stabilization of AIMP2-DX2. AIMP2-DX2 inhibits p14/ARF by directly binding to it, thereby suppressing oncogene-induced apoptosis and senescence. AIMP2, ARS-interacting multifunctional protein 2; AIMP2-DX2, AIMP2 lacking exon 2; MSC, multi-tRNA synthetase complex; MAPK, mitogen-activated protein kinase; Smurf2, Smad ubiquitin regulatory factors 2; FBP, FUSE-binding protein; CRM1, chromosomal region maintenance 1; TGF- $\beta$, transforming growth factor- $\beta$; HSP70, heat shock protein 70; Siah1, Seven in absentia homolog 1.

nuclear output of Smurf2 (Kim et al., 2016). Notably, the A549 cells expressing AIMP2 S156A mutant were more likely to generate tumors in vivo, indicating that AIMP2 played an essential role in regulating the growth inhibitory activity of TGF- $\beta$ (Kim et al., 2016). Lim et al. (2020) revealed that the levels of heat shock protein 70 (HSP70) and AIMP2-DX2 were positively correlated in lung cancer cells, and HSP70 could regulate AIMP2-DX2 level to some extent. Specifically, HSP70 inhibited the Seven in absentia homolog 1 (Siah1)-dependent AIMP2-DX2 ubiquitination by interacting with AIMP2-DX2, thereby maintaining the stabilization of AIMP2-DX2 (Lim et al., 2020). Meaningfully, a compound BC-DXI-495 blocked the interaction of HSP70 with AIMP2-DX2 and thus inhibited cancer progression in vitro and in vivo (Lim et al., 2020). Moreover, AIMP2-DX2 inhibited p14/ARF by directly binding to it, thereby suppressing oncogene-induced apoptosis and senescence (Oh et al., 2016). SLCB050 prevented their interaction and inhibited the development of lung cancer induced by ectopic expression of AIMP2-DX2 in mice, which provided a new idea for cancer treatment (Oh et al., 2016).

\section{ARSs and Breast Cancer}

Breast cancer is the most common malignancy in women, which threatens the lives of patients and seriously affects their quality of life (Au et al., 2020). Recent studies discovered that four single nucleotide polymorphisms (SNPs) in ARS genes, including rs34087264 in alanyl-tRNA synthetase (AlaRS), rs801186 in histidyl-tRNA Synthetase (HARS), rs193466 in arginyl-tRNA synthetase (RARS) and rs2273802 in tryptophanyltRNA synthetase (WARS), were significantly associated with an increased risk of breast cancer in Chinese population (He et al., 2015). Accumulating evidence has implicated the insulinlike growth factor (IGF) signaling with breast cancer biology (Ireland et al., 2018; De Vincenzo et al., 2019). IGF overexpression significantly promoted cell proliferation and tumor formation in mice (Pacher et al., 2007). Notably, MRS was up-regulated in the MCF7 cells stably overexpressing IGF1 or IGF2, indicating that MRS might mediate the tumorigenesis regulated by IGF (Pacher et al., 2007). It is well known that the activity of cyclin-dependent kinase 4 (CDK4) is inhibited by p16 ${ }^{\text {INK4a }}$ (Cen et al., 2012; Liao et al., 2020). Intriguingly, MRS maintained the stability of CDK4 by interacting with CDK4 and heat shock protein 90 (HSP90), thereby regulating the cell cycle (Kwon et al., 2018). Since MRS could compete with $\mathrm{p} 16^{\mathrm{INK} 4 \mathrm{a}}$ for binding to CDK4, the stabilizing effect of MRS on CDK4 was more significant in $\mathrm{p}^{\mathrm{INK} 4 \mathrm{a}_{\text {- }}}$ negative breast cancer cells (Kwon et al., 2018). Suppression of MRS reduced the tumorigenic ability of MDA-MB-231 cells in vivo, implying that MRS was involved in the development of breast cancer (Kwon et al., 2018). Furthermore, Qi et al. (2019) identified 9 differentially expressed-methylated hub genes in breast cancer. Of these, glutamyl-prolyl-tRNA synthetase (EPRS) was up-regulated and was related to poor clinical outcomes 
in breast cancer, suggesting that EPRS might be regulated by DNA methylation and contributed to the development of breast cancer (Qi et al., 2019). Similarly, EPRS was found to be highly expressed in estrogen receptor positive $(\mathrm{ER}+)$ breast cancer tissues and was associated with reduced overall survival in TCGA and METABRIC datasets (Katsyv et al., 2016). Further studies discovered that EPRS regulated cell cycle and estrogen response genes, indicating that EPRS might serve as an important regulator of cell proliferation and estrogen signaling in ER+ breast cancer (Katsyv et al., 2016). In addition, AIMP1 significantly inhibited the growth of breast cancer 4T1 cells in mice, accompanied by decreased myeloid-derived suppressor cell (MDSC) population in the spleens and tumor sites (Hong et al., 2016). Meanwhile, AIMP1 not only suppressed the expansion of MDSCs in vitro, but also reduced the expression of IL-6, NO and arginase- 1 in MDSCs (Hong et al., 2016). In-depth studies found that AIMP1 inhibited the expansion and suppressive functions of MDSCs by reducing the activity of signal transducers and activators of transcription (STATs), AKT and ERK, indicating that AIMP1 might be a key target of breast cancer immunotherapy strategies (Hong et al., 2016). Strikingly, RARS overexpression significantly reduced AIMP1 secretion in HeLa and MCF7 cells, indicating that RARS might participate in tumorigenesis by regulating the secretion of AIMP1 in cancer cells (Bottoni et al., 2007).

\section{ARSs and Other Cancers}

Gastric cancer is the fourth most common malignant tumor, characterized by high mortality, low rates of early diagnosis and poor prognosis (Shafabakhsh et al., 2020). KRS was highly expressed in gastric cancer tissues and tumor-associated inflammatory cells (Kim B.H. et al., 2014). Meaningfully, the high levels of KRS in tumor cells were associated with shorter overall survival, while its high levels in tumor-associated inflammatory cells were associated with longer overall survival, indicating that KRS might act as a potential biomarker for gastric cancer (Kim B.H. et al., 2014). Analogously, another research found that both WARS and indoleamine 2,3-dioxygenase 1 (IDO1) were highly expressed in Epstein-Barr virus-associated and microsatellite instability-high subtypes of gastric cancer (Lu et al., 2020). The expression of WARS and IDO1 was related to poor prognosis in p53-aberrant and p53-wildtype subtypes, while the expression of WARS was related to better prognosis in microsatellite instability subtype (Lu et al., 2020). Tian et al. (2017) demonstrated that four SNPs in cysteinyl-tRNA synthetase (CARS), including rs384490, rs7394702, rs2071101, and rs729662, were associated with gastric cancer risk. Of these, rs384490 and rs7394702 were found to alter the DNA methylation or transcription factor response elements of CARS by silico analysis, and rs384490 and rs729662 were found to regulate the expression of CARS-related genes by expression quantitative trait loci analysis, indicating that CARS might be involved in the development of gastric cancer (Tian et al., 2017). Moreover, TyrRS knockdown significantly inhibited the proliferation of gastric cancer cell lines AGS, HGC-27 and MGC-803, while TyrRS overexpression promoted the tumor growth in HGC-27 xenograft models (Zhang et al., 2020). Further studies found that TyrRS could facilitate gastric cancer progression and homologous recombination process by regulating phosphatidylinositol 3-kinase (PI3K)/AKT signaling (Zhang et al., 2020). Melanoma is a type of aggressive skin cancer with high heterogeneity (Pierce and Simmons, 2020). WARS could regulate the proliferation, migration and invasion of uveal melanoma cells via inducing the activation of PI3K/AKT/mTOR pathway, suggesting that WARS might be is a novel tumorigenic factor (Yang et al., 2020). Liang et al. (2017) observed that AIMP1 was essential for the bone marrow-derived dendritic cell (BMDC) vaccine-mediated protection against melanoma. In detail, AIMP1 enhanced the T-helper type $1\left(\mathrm{~T}_{\mathrm{H}} 1\right)$ polarization partly through p38/MAPK signaling pathway, thereby exerting an antitumor effect (Liang et al., 2017).

Furthermore, ARSs were also associated with other cancers, such as liver cancer, pancreatic cancer, leukemia and oral cancer (Chen et al., 2011; Paley et al., 2011; Ma et al., 2013; Lee et al., 2015). Previous studies found that WARS and fumarate hydratase were involved in the hepatitis B virus (HBV)induced angiogenesis in rat primary hepatocytes and HepG2 cells by LC-MS/MS analysis, indicating that these two proteins might act as potential anti-angiogenic targets in liver cancer therapy (Zhang et al., 2009). Nuclear transcription factor nuclear factor of activated $\mathrm{T}$ cells 5 (NFAT5) has been implicated in the pathogenesis of various human cancers (Jiang et al., 2019; Liu et al., 2020). Interestingly, HBV enhanced liver cancer progression by suppressing NFAT5 (Qin et al., 2017). In this process, HBV not only inhibited NFAT5 expression by inducing the hypermethylation of NFAT5 promoter, but also inhibited NFAT5 through MAPK activated by miR-30e-5p (Qin et al., 2017). Subsequently, the low expression of NFAT5 could up-regulate aspartyl-tRNA synthetase 2 (DARS2), thereby promoting liver cancer development by accelerating cell cycle progression and inhibiting cell apoptosis. MUC1, a member of the mucin family, is closely associated with cancer pathology (Kufe, 2009). Knockdown of threonyl-tRNA synthetase (TRS) could reduce the level of MUC1 and thus inhibit the migration of pancreatic cancer cells (Jeong et al., 2018). Moreover, IARS2 knockdown increased the levels of p53 and p21 and decreased the levels of proliferating cell nuclear antigen (PCNA) and eukaryotic translation initiation factor $4 \mathrm{E}$ (eIF4E) in HL-60 cells, thereby inhibiting cell proliferation ( $\mathrm{Li} \mathrm{H}$. et al., 2019). These results indicate that IARS2 may participate in the development of acute myeloid leukemia by regulating the p53/p21/PCNA/eIF4E pathway. Besides from the tight correlation with the high relapse-free survival of breast cancer patients, overexpression of SerRS also suppressed the growth of cervical tumor xenografts by inducing cellular senescence in vivo, indicating that SerRS might have a certain degree of antitumor activity (Li Y. et al., 2019). Mechanistically, SerRS interacted with the telomere DNA repeats and recruited more protection of telomeres 1 (POT1) proteins to telomeres in the nucleus, thereby blocking telomerase recruitment and telomere elongation (Li Y. et al., 2019). These results indicate that SerRS may inhibit tumorigenesis by inducing cellular senescence. Mo et al. (2016) discovered that glycyl-tRNA synthetase (GRS) had the strongest correlation with mortality by analyzing the expression of 20 ARSs in 3557 breast cancer patients. Neddylation is a post-translational modification that attaches ubiquitin-like protein NEDD8 to its substrates and is 
implicated in cancer progression (Heo et al., 2020; Zhou X. et al., 2020). In particular, GRS captured and protected NEDD8conjugated Ubc12 (activated E2) by binding to the APPBP1 subunit of E1, thereby increasing the global level of neddylation (Mo et al., 2016). Indeed, knockdown of GRS decreased the levels of NEDD8-conjugated Ubc12 and cullin neddylation in HeLa cells, leading to cell cycle arrest (Mo et al., 2016).

\section{POTENTIAL CLINICAL APPLICATIONS OF ARSs IN CANCER}

\section{ARSs as Potential Biomarkers}

Meaningfully, certain ARSs can be used as diagnostic and prognostic biomarkers for cancer patients (Table 1). The levels of NRS and ERO1-like protein alpha (ERO1L) were higher in lung adenocarcinoma tissues compared with neighboring normal tissues, and their levels were positively associated with lymph node metastasis (Hsu et al., 2016). At the same time, ERO1L overexpression was associated with the poor survival rate of patients with early adenocarcinoma. Importantly, NARS or ERO1L knockdown could inhibit the growth and migration of adenocarcinoma cells (Hsu et al., 2016). These findings indicate that NRS and ERO1L not only play a role in cancer progression, but may also serve as novel markers for lung adenocarcinoma. Kim E.Y. et al. (2017) found that MRS was frequently overexpressed in cancer tissues from LSLKras G12D and LSL-Kras G12D:p53 ${ }^{\mathrm{fl} / \mathrm{fl}}$ mice and human lung cancer tissues. Importantly, MRS could regulate the activity of mTORC1 signaling, and its overexpression was associated with poor prognosis in NSCLC, suggesting that MRS might contribute to the development of lung cancer (Kim E.Y. et al., 2017). Furthermore, the levels of nuclear KRS were significantly predictive of disease-free survival in NSCLC patients (Boulos et al., 2017).

Additionally, the plasma KRS levels of patients with colorectal cancer were higher than those of healthy controls, and the levels were significantly reduced after surgery (Suh et al., 2020). Meanwhile, KRS levels were highly correlated with the number of polyps in the colorectal cancer mouse model. Notably, KRS had a good diagnostic value for colorectal cancer, and its diagnostic capability and specificity were better than those of current biomarkers, including carcinoembryonic antigen (CEA) and carbohydrate antigen 19-9 (CA19-9) (Suh et al., 2020). TRS levels in human ovarian cancer tissues were positively correlated with disease stage and vascular endothelial growth factor (VEGF) levels (Wellman et al., 2014). TRS could be secreted by cancer cells as a cellular stress response (Wellman et al., 2014). The serum TRS levels of patients were associated with tumor TRS and VEGF, but not with disease stage. These data indicate that TRS may be involved in tumor angiogenesis and serve as a potential biomarker for the diagnosis of ovarian cancer. Intriguingly, AIMP3 expression was reduced in muscleinvasive bladder cancer (MIBC) and was associated with genomic instability and radiation resistance (Gurung et al., 2015). AIMP3 levels could predict the overall survival following radiotherapy and disease recurrence in MIBC patients, which provided new ideas for the radiotherapy or chemo-radiotherapy of bladder cancer (Gurung et al., 2015).

\section{ARSs and Therapeutic Applications}

Since ARSs are closely related to tumor development, they may serve as potential targets for cancer treatment (Figure 4). LRS was often overexpressed in lung cancer tissues and performed an important function in the activation of mTORC1 and cell growth (Kim et al., 2019). The novel LRS inhibitor BC-LI-0186 could inhibit mTORC1 signaling, resulting in cytotoxicity to NSCLC cells and anticancer effects in K-ras mouse lung cancer model (Kim et al., 2019). In this process, BC-LI-0186 bound to the RagD interacting site of LRS, thus suppressing the lysosomal localization and activity of mTORC1 (Kim J.H. et al., 2017). Beltran et al. (2014) observed that the engineering interference peptides targeting Engrailed 1 (EN1) induced apoptosis of cancer cells and increased the sensitivity of resistant breast cancer cells to chemotherapy drugs by blocking the function of EN1. Interestingly, these interference peptides could also regulate downstream EPRS effectors by binding to EPRS in SUM149PT cells, indicating that EPRS inhibition might act as a potential treatment for breast cancer. Furthermore, BC-DXI-843, a sulfonamide based AIMP2-DX2 inhibitor, promoted the degradation of AIMP2DX2 by blocking the interaction between AIMP2-DX2 and HSP70, thereby inducing cancer cell apoptosis (Sivaraman et al., 2020). Of note, BC-DXI-843 effectively reduced the tumorigenicity of $\mathrm{H} 460$ cells in mice (Sivaraman et al., 2020). Similarly, aminophenylpyrimidine 3 could exert anti-tumor

TABLE 1 | Aminoacyl-tRNA synthetases (ARSs) as potential biomarkers in cancer.

\begin{tabular}{|c|c|c|c|c|}
\hline ARSs & Cancer type & Expression status & Effects & References \\
\hline AIMP2-DX2 & Lung cancer & Upregulation & The ratio of AIMP2-DX2 to AIMP2 was negatively correlated with patient survival. & Choi et al., 2011 \\
\hline EPRS & Breast cancer & Upregulation & Related to poor clinical outcomes in breast cancer. & Qi et al., 2019 \\
\hline SerRS & Breast cancer & Downregulation & High levels of SerRS were tightly correlated with the high relapse-free survival. & Li Y. et al., 2019 \\
\hline NRS & Lung cancer & Upregulation & Positively associated with lymph node metastasis. & Hsu et al., 2016 \\
\hline MRS & Lung cancer & Upregulation & Associated with poor prognosis in NSCLC. & Kim E.Y. et al., 2017 \\
\hline KRS & Lung cancer & Upregulation & Predicted disease-free survival in NSCLC patients. & Boulos et al., 2017 \\
\hline KRS & Colon cancer & Upregulation & Had a good diagnostic value for colorectal cancer. & Suh et al., 2020 \\
\hline TRS & Ovarian cancer & Upregulation & Positively correlated with disease stage and VEGF levels. & Wellman et al., 2014 \\
\hline AIMP3 & Bladder cancer & Downregulation & Predicted the overall survival following radiotherapy and disease recurrence in MIBC patients. & Gurung et al., 2015 \\
\hline
\end{tabular}


A

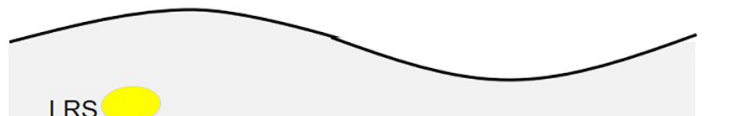

LRS

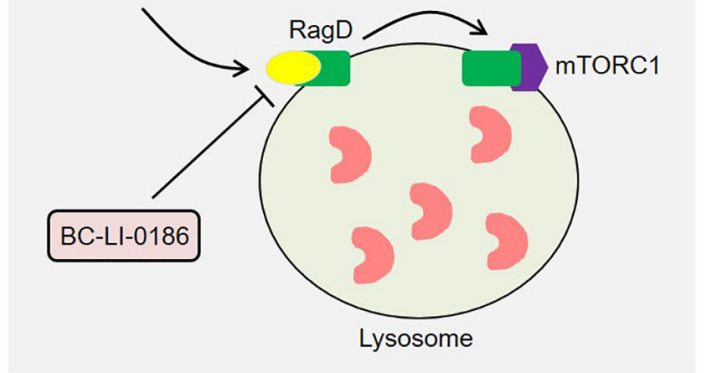

c

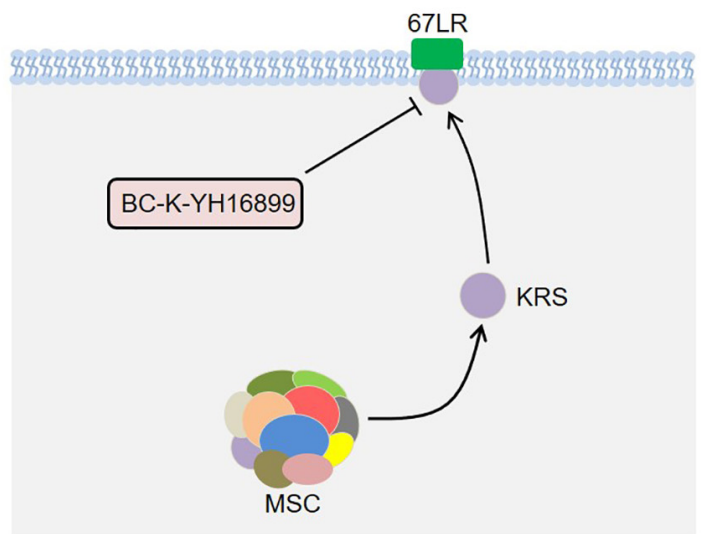

E

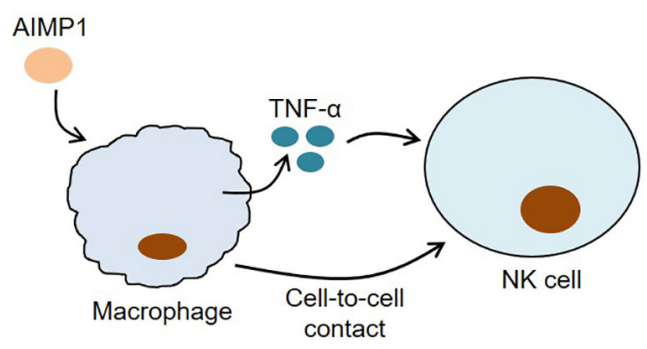

B

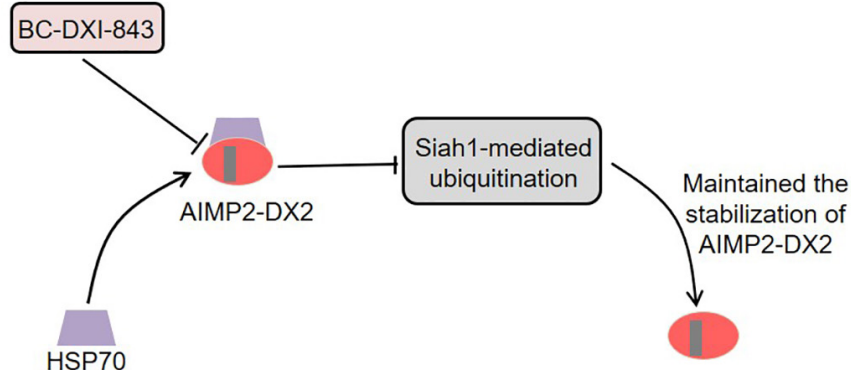

D

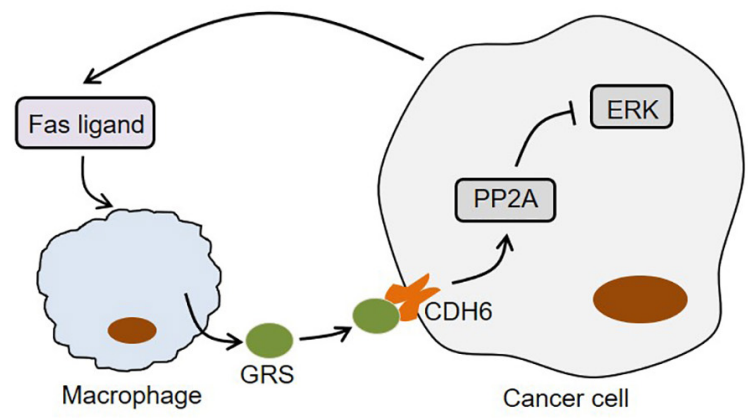

F

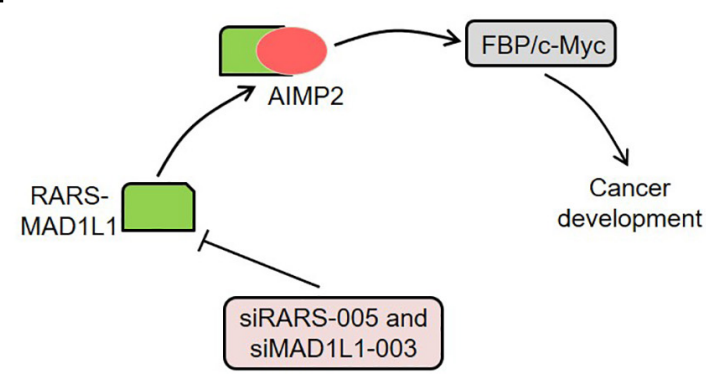

FIGURE 4 | Aminoacyl-tRNA synthetases and potential therapeutic applications. (A) BC-LI-0186 binds to the RagD interacting site of LRS, thus suppressing the Iysosomal localization and activity of mTORC1. (B) BC-DXI-843 promotes the degradation of AIMP2-DX2 by blocking the interaction between AIMP2-DX2 and HSP70, thereby inducing cancer cell apoptosis. (C) BC-K-YH16899 not only blocks the interaction of KRS and 67LR by binding to KRS, but also reduces the KRS membrane localization by restraining the flexible N-ext of KRS. (D) Macrophages secretes GRS under the stimulation of Fas ligand released by tumor cells. The secreted GRS interacts with specific ERK-activated tumor cells through CDH6, thereby enhancing PP2A activity by releasing PP2A from CDH6. The activated PP2A inhibits ERK signaling by binding to ERK and thus induces apoptosis of cancer cells. (E) AIMP1 inhibits the lung metastasis of melanoma cells in mice through macrophage-mediated activation of NK cells. AIMP1 induces macrophages to produce TNF- $\alpha$, which partially activates NK cells. Meanwhile, the activation of NK cells requires the direct contact between NK cells and macrophages. (F) The knockdown of RARS-MAD1L1 using siRARS-005 or siMAD1L1-003 inhibits cancer development by suppressing FBP/c-Myc pathway. ARSs, aminoacyl-tRNA synthetases; LRS, leucyl-tRNA synthetase; mTORC1, mammalian target of rapamycin complex 1; AIMP2-DX2, AIMP2 lacking exon 2; HSP70, heat shock protein 70; KRS, lysyl-tRNA synthetase; 67LR, 67-kDa laminin receptor; GRS, glycyl-tRNA synthetase; CDH6, cadherin-6; PP2A, phosphatase 2A; ERK, extracellular signal-regulated kinase; AIMP1, ARS-interacting multifunctional protein 1; TNF- $\alpha$, tumor necrosis factor $\alpha$; NK cell, natural killer cell; RARS, arginyl-tRNA synthetase; MAD1L1, mitotic arrest deficient 1-like 1; FBP, FUSE-binding protein.

effects by inhibiting AIMP2-DX2 in H460 and A549 cells (Lee et al., 2020).

Compound BC-K-YH16899 inhibited KRS-mediated cancer metastasis in vivo without affecting the catalytic activity of KRS (Kim D.G. et al., 2014). Specifically, BC-K-YH16899 not only blocked the interaction of KRS and 67LR by binding to KRS, but also reduced the KRS membrane localization by restraining the flexible N-ext of KRS. These findings indicate the potential of KRS as a target for antimetastatic therapy (Kim D.G. et al., 2014). In fact, the molecular structure of some ARSs contains a zinc atom, a sulfonamide binding scaffold, which provides an idea for the development of ARS inhibitors 
(Sankaranarayanan et al., 2000; Bilokapic et al., 2006; Kumar et al., 2015). Strikingly, macrophages secreted GRS under the stimulation of Fas ligand released by tumor cells (Park et al., 2012). The secreted GRS interacted with specific ERK-activated tumor cells through cadherin-6 (CDH6), thereby enhancing phosphatase 2A (PP2A) activity by releasing PP2A from CDH6 and reducing PP2A phosphorylation (Park et al., 2012). Next, the activated PP2A inhibited ERK signaling by binding to ERK and reducing its phosphorylation, thus inducing apoptosis of cancer cells. Meaningfully, GRS significantly suppressed tumor growth in a xenograft model using HCT116 cells, indicating that GRS might be a potential target for cancer treatment (Park et al., 2012). Furthermore, AIMP1 could inhibit the lung metastasis of melanoma cells in mice through macrophagemediated activation of natural killer (NK) cells (Kim M.S. et al., 2017). On the one hand, AIMP1 significantly induced macrophages to produce TNF- $\alpha$, which partially activated NK cells (Kim M.S. et al., 2017). On the other hand, the activation of NK cells induced by AIMP1 required the direct contact between NK cells and macrophages. The fusion protein RARS-mitotic arrest deficient 1-like 1 (MAD1L1) activated the FBP/c-Myc pathway by interacting with AIMP2, thereby inducing cancer stem cell-like phenotypes (Zhong et al., 2018). The knockdown of RARS-MAD1L1 reduced the growth and colony formation of cancer cells in vitro, indicating that RARS-MAD1L1 inhibition might be a new strategy for cancer treatment (Zhong et al., 2018).

\section{CONCLUSION AND FUTURE PERSPECTIVE}

Traditionally, ARSs are considered as housekeeping molecules that mainly participate in protein synthesis by catalyzing the aminoacylation of tRNAs. Therefore, these enzymes are necessary for maintaining cell homeostasis and normal physiological functions of the body. In fact, in addition to this main function, ARSs also play an important role in various pathological processes (Stephen et al., 2018; D’Hulst et al., 2020; Wu et al., 2020). Strikingly, ARSs are closely associated with tumor biology. On the one hand, ARSs are involved in the progression of various cancers, such as colon cancer, lung cancer, breast cancer, gastric cancer and pancreatic cancer. Among them, some ARSs such as IARS2, KRS and NRS have been reported to promote the development of cancer (Kim et al., 2012; Fang et al., 2018; Yeom

\section{REFERENCES}

Akaike, T., Ida, T., Wei, F. Y., Nishida, M., Kumagai, Y., Alam, M. M., et al. (2017). Cysteinyl-tRNA synthetase governs cysteine polysulfidation and mitochondrial bioenergetics. Nat. Commun. 8:1177. doi: 10.1038/s41467-017-01311-y

Antonellis, A., and Green, E. D. (2008). The role of aminoacyl-tRNA synthetases in genetic diseases. Annu. Rev. Genom. Hum. Genet. 9, 87-107. doi: 10.1146/ annurev.genom.9.081307.164204

Au, C. C., Furness, J. B., Britt, K., Oshchepkova, S., Ladumor, H., Soo, K. Y., et al. (2020). Three-dimensional growth of breast cancer cells potentiates the antitumor effects of unacylated ghrelin and AZP-531. eLife 9:e56913. doi: 10.7554/ eLife. 56913 et al., 2020), while AIMPs, SerRS and GRS usually exert antitumor effects (Park et al., 2012; Li Y. et al., 2019; Zhou Z. et al., 2020). On the other hand, ARSs can be used not only as biomarkers for cancer diagnosis and prognosis, but also as potential targets for inhibiting cancer growth and metastasis.

Aminoacyl-tRNA synthetases have evolutionarily conserved enzymatic mechanisms, and the understanding of their molecular structure is conducive to the rational design of drugs. Indeed, the therapeutic applications of ARSs have begun to be explored in human diseases, especially in infections, cancer and autoimmune diseases (Keller et al., 2012; Baragana et al., 2019; Kwon et al., 2019). Since ARSs are directly involved in protein synthesis, we should pay more attention to the possible cytotoxicity when studying the potential applications. As described in the article, ARSs and AIMPs promote or inhibit cancer development through different molecular mechanisms. Therefore, a variety of treatment modalities targeting ARSs can be explored, such as compound inhibitors, peptides and gene therapy. Considering that amino acids are the substrates of ARSs, the involvement of high-protein diets or ketogenic diets in the development of tumors through regulating ARSs may be an interesting research direction. Moreover, some types of cancer are described to depend on specific amino acids (Tang et al., 2017; Salzer et al., 2018), and certain ARSs can regulate amino acid transport (Moore et al., 1977; Miyanokoshi et al., 2018). Therefore, further studies are needed to determine whether ARSs influence cancer development by regulating amino acid transporters. All in all, we summarized the roles of ARSs in tumorigenesis, with the purpose of providing new ideas for cancer treatment.

\section{AUTHOR CONTRIBUTIONS}

ZZ and BS contributed toward the concept and manuscript writing. AN participated in the literature search and discussion. DY and MB revised and supervised overall project. All authors read and approved the final version of manuscript.

\section{ACKNOWLEDGMENTS}

The authors wish to acknowledge Xiaochuan Zhang from the First Affiliated Hospital of Zhengzhou University, China for editing of English grammar and syntax of the manuscript.

Baragana, B., Forte, B., Choi, R., Nakazawa Hewitt, S., Bueren-Calabuig, J. A., and Pisco, J. P. (2019). Lysyl-tRNA synthetase as a drug target in malaria and cryptosporidiosis. Proc. Natl. Acad. Sci. U.S.A. 116, 7015-7020. doi: 10.1073/ pnas. 1814685116

Beltran, A. S., Graves, L. M., and Blancafort, P. (2014). Novel role of Engrailed 1 as a prosurvival transcription factor in basal-like breast cancer and engineering of interference peptides block its oncogenic function. Oncogene 33, 4767-4777. doi: 10.1038/onc.2013.422

Bilokapic, S., Maier, T., Ahel, D., Gruic-Sovulj, I., Söll, D., Weygand-Durasevic, I., et al. (2006). Structure of the unusual seryl-tRNA synthetase reveals a distinct zinc-dependent mode of substrate recognition. EMBO J. 25, 2498-2509. doi: 10.1038/sj.emboj.7601129 
Bin, J., Nie, S., Tang, Z., Kang, A., Fu, Z., Hu, Y., et al. (2020). Long noncoding RNA EPB41L4A-AS1 functions as an oncogene by regulating the Rho/ROCK pathway in colorectal cancer. J. Cell. Physiol. doi: 10.1002/jcp.29880 [Epub ahead of print].

Bottoni, A., Vignali, C., Piccin, D., Tagliati, F., Luchin, A., Zatelli, M. C., et al. (2007). Proteasomes and RARS modulate AIMP1/EMAP II secretion in human cancer cell lines. J. Cell Physiol. 212, 293-297. doi: 10.1002/jcp.21083

Boulos, S., Park, M. C., Zeibak, M., Foo, S. Y., Jeon, Y. K., Kim, Y. T., et al. (2017). Serine 207 phosphorylated lysyl-tRNA synthetase predicts disease-free survival of non-small-cell lung carcinoma. Oncotarget 8, 65186-65198. doi: 10.18632/oncotarget.18053

Cen, L., Carlson, B. L., Schroeder, M. A., Ostrem, J. L., Kitange, G. J., Mladek, A. C., et al. (2012). p16-Cdk4-Rb axis controls sensitivity to a cyclin-dependent kinase inhibitor PD0332991 in glioblastoma xenograft cells. Neuro Oncol. 14, 870-881. doi: 10.1093/neuonc/nos114

Chen, S. H., Yang, W., Fan, Y., Stocco, G., Crews, K. R., Yang, J. J., et al. (2011). A genome-wide approach identifies that the aspartate metabolism pathway contributes to asparaginase sensitivity. Leukemia 25, 66-74. doi: 10.1038/leu. 2010.256

Choi, J. W., Kim, D. G., Lee, A. E., Kim, H. R., Lee, J. Y., Kwon, N. H., et al. (2011). Cancer-associated splicing variant of tumor suppressor AIMP2/p38: pathological implication in tumorigenesis. PLoS Genet. 7:e1001351. doi: 10. 1371/journal.pgen.1001351

Choi, J. W., Um, J. Y., Kundu, J. K., Surh, Y. J., and Kim, S. (2009). Multidirectional tumor-suppressive activity of AIMP2/p38 and the enhanced susceptibility of AIMP2 heterozygous mice to carcinogenesis. Carcinogenesis 30, 1638-1644. doi: $10.1093 /$ carcin/bgp 170

De Vincenzo, A., Belli, S., Franco, P., Telesca, M., and Iaccarino, I. (2019). Paracrine recruitment and activation of fibroblasts by c-Myc expressing breast epithelial cells through the IGFs/IGF-1R axis. Science 145, 2827-2839. doi: 10.1002/ijc. 32613

D'Hulst, G., Soro-Arnaiz, I., Masschelein, E., and Veys, K. (2020). PHD1 controls muscle mTORC1 in a hydroxylation-independent manner by stabilizing leucyl tRNA synthetase. Nat. Commun. 11:174. doi: 10.1038/s41467-019-13889-6

Di, X., Jin, X., Ma, H., Wang, R., Cong, S., Tian, C., et al. (2019). The oncogene IARS2 promotes non-small cell lung cancer tumorigenesis by activating the AKT/MTOR pathway. Front. Oncol. 9:393. doi: 10.3389/fonc.2019.00393

Drula, R., Braicu, C., Harangus, A., Nabavi, S. M., Trif, M., Slaby, O., et al. (2020). Critical function of circular RNAs in lung cancer. Wiley Interdiscip. Rev. RNA 11:e1592. doi: 10.1002/wrna.1592

Fang, Z., Wang, X., Yan, Q., Zhang, S., and Li, Y. (2018). Knockdown of IARS2 suppressed growth of gastric cancer cells by regulating the phosphorylation of cell cycle-related proteins. Mol. Cell Biochem. 443, 93-100. doi: 10.1007/s11010017-3213-8

Fu, C. Y., Wang, P. C., and Tsai, H. J. (2017). Competitive binding between SeryltRNA synthetase/YY1 complex and NFKB1 at the distal segment results in differential regulation of human vegfa promoter activity during angiogenesis. Nucleic Acids Res. 45, 2423-2437. doi: 10.1093/nar/gkw1187

Gao, W., An, C., Xue, X., Zheng, X., Niu, M., Zhang, Y., et al. (2019). Mass spectrometric analysis identifies AIMP1 and LTA4H as FSCN1-binding proteins in laryngeal squamous cell carcinoma. Proteomics 19:e1900059. doi: 10.1002/pmic.201900059

Gurung, P. M., Veerakumarasivam, A., Williamson, M., Counsell, N., Douglas, J., Tan, W. S., et al. (2015). Loss of expression of the tumour suppressor gene AIMP3 predicts survival following radiotherapy in muscle-invasive bladder cancer. Int. J. Cancer 136, 709-720. doi: 10.1002/ijc.29022

He, X. D., Gong, W., Zhang, J. N., Nie, J., Yao, C. F., Guo, F. S., et al. (2018). Sensing and transmitting intracellular amino acid signals through reversible lysine aminoacylations. Cell Metab. 27, 151-166.e156. doi: 10.1016/j.cmet.2017.10.015

He, Y., Gong, J., Wang, Y., Qin, Z., Jiang, Y., Ma, H., et al. (2015). Potentially functional polymorphisms in aminoacyl-tRNA synthetases genes are associated with breast cancer risk in a Chinese population. Mol. Carcinog. 54, 577-583. doi: $10.1002 / \mathrm{mc} .22128$

Heo, M. J., Kang, S. H., Kim, Y. S., Lee, J. M., Yu, J., Kim, H. R., et al. (2020). UBC12-mediated SREBP-1 neddylation worsens metastatic tumor prognosis. Mol. Cancer Biol. 147, 2550-2563. doi: 10.1002/ijc.33113

Hong, H. J., Lim, H. X., Song, J. H., Lee, A., Kim, E., Cho, D., et al. (2016). Aminoacyl-tRNA synthetase-interacting multifunctional protein 1 suppresses tumor growth in breast cancer-bearing mice by negatively regulating myeloidderived suppressor cell functions. Cancer Immunol. Immunother. 65, 61-72. doi: 10.1007/s00262-015-1777-2

Hsu, C. H., Hsu, C. W., Hsueh, C., Wang, C. L., Wu, Y. C., Wu, C. C., et al. (2016). Identification and characterization of potential biomarkers by quantitative tissue proteomics of primary lung adenocarcinoma. Mol. Cell Proteom. 15, 2396-2410. doi: 10.1074/mcp.M115.057026

Hyeon, D. Y., Kim, J. H., Ahn, T. J., and Cho, Y. (2019). Evolution of the multitRNA synthetase complex and its role in cancer. J. Biol. Chem. 294, 5340-5351. doi: 10.1074/jbc.REV118.002958

Ireland, L., Santos, A., Campbell, F., Figueiredo, C., Hammond, D., Ellies, L. G., et al. (2018). Blockade of insulin-like growth factors increases efficacy of paclitaxel in metastatic breast cancer. Oncogene 37, 2022-2036. doi: 10.1038/ s41388-017-0115-x

Jeong, S. J., Kim, J. H., Lim, B. J., Yoon, I., Song, J. A., Moon, H. S., et al. (2018). Inhibition of MUC1 biosynthesis via threonyl-tRNA synthetase suppresses pancreatic cancer cell migration. Exp. Mol. Med. 50:e424. doi: 10.1038/emm. 2017.231

Jiang, Y., He, R., Jiang, Y., Liu, D., Tao, L., Yang, M., et al. (2019). Transcription factor NFAT5 contributes to the glycolytic phenotype rewiring and pancreatic cancer progression via transcription of PGK1. Cell 10:948. doi: 10.1038/s41419019-2072-5

Jung, J. Y., Kim, E. Y., Kim, A., Chang, J., Kwon, N. H., Moon, Y., et al. (2017). Ratio of autoantibodies of tumor suppressor AIMP2 and its oncogenic variant is associated with clinical outcome in lung cancer. J. Cancer 8, 1347-1354. doi: $10.7150 /$ jca. 18450

Katsyv, I., Wang, M., Song, W. M., Zhou, X., Zhao, Y., Park, S., et al. (2016). EPRS is a critical regulator of cell proliferation and estrogen signaling in ER+ breast cancer. Oncotarget 7, 69592-69605. doi: 10.18632/oncotarget.11870

Keller, T. L., Zocco, D., Sundrud, M. S., Hendrick, M., Edenius, M., Yum, J., et al. (2012). Halofuginone and other febrifugine derivatives inhibit prolyl-tRNA synthetase. Nat. Chem. Biol. 8, 311-317. doi: 10.1038/nchembio.790

Kim, B. H., Jung, W. Y., Lee, H., Kang, Y., Jang, Y. J., Hong, S. W., et al. (2014). Lysyl-tRNA synthetase (KRS) expression in gastric carcinoma and tumor-associated inflammation. Ann. Surg. Oncol. 21, 2020-2027. doi: 10.1245/ s10434-014-3522-z

Kim, D. G., Lee, J. Y., Kwon, N. H., Fang, P., Zhang, Q., Wang, J., et al. (2014). Chemical inhibition of prometastatic lysyl-tRNA synthetase-laminin receptor interaction. Nat. Chem. Biol. 10, 29-34. doi: 10.1038/nchembio.1381

Kim, D. G., Choi, J. W., Lee, J. Y., Kim, H., Oh, Y. S., Lee, J. W., et al. (2012). Interaction of two translational components, lysyl-tRNA synthetase and p40/37LRP, in plasma membrane promotes laminin-dependent cell migration. FASEB J. 26, 4142-4159. doi: 10.1096/fj.12-207639

Kim, D. G., Lee, J. Y., Lee, J. H., Cho, H. Y., Kang, B. S., Jang, S. Y., et al. (2016). Oncogenic mutation of AIMP2/p38 inhibits its tumor-suppressive interaction with Smurf2. Cancer Res. 76, 3422-3436. doi: 10.1158/0008-5472.can-153255

Kim, E. Y., Jung, J. Y., Kim, A., Kim, K., and Chang, Y. S. (2017). MethionyltRNA synthetase overexpression is associated with poor clinical outcomes in non-small cell lung cancer. BMC Cancer 17:467. doi: 10.1186/s12885-0173452-9

Kim, J. H., Lee, C., Lee, M., Wang, H., Kim, K., Park, S. J., et al. (2017). Control of leucine-dependent mTORC1 pathway through chemical intervention of leucyltRNA synthetase and RagD interaction. Nat. Commun. 8:732. doi: 10.1038/ s41467-017-00785-0

Kim, M. S., Song, J. H., Cohen, E. P., Cho, D., and Kim, T. S. (2017). Aminoacyl tRNA synthetase-interacting multifunctional protein 1 activates NK cells via macrophages in vitro and in vivo. J. Immunol. 198, 4140-4147. doi: 10.4049/ jimmunol.1601558

Kim, S. B., Kim, H. R., Park, M. C., Cho, S., and Goughnour, P. C. (2017). Caspase-8 controls the secretion of inflammatory lysyl-tRNA synthetase in exosomes from cancer cells. J. Cell Biol. 216, 2201-2216. doi: 10.1083/jcb.201605118

Kim, E. Y., Lee, J. G., Lee, J. M., Kim, A., Yoo, H. C., Kim, K., et al. (2019). Therapeutic effects of the novel Leucyl-tRNA synthetase inhibitor BC-LI-0186 in non-small cell lung cancer. Ther. Adv. Med. Oncol. 11:1758835919846798. doi: $10.1177 / 1758835919846798$

Kufe, D. W. (2009). Mucins in cancer: function, prognosis and therapy. Nat. Rev. Cancer 9, 874-885. doi: 10.1038/nrc2761 
Kumar, M., Kumar, S. A., Dimkovikj, A., Baykal, L. N., Banton, M. J., Outlaw, M. M., et al. (2015). Zinc is the molecular "switch" that controls the catalytic cycle of bacterial leucyl-tRNA synthetase. J. Inorg. Biochem. 142, 59-67. doi: 10.1016/j.jinorgbio.2014.09.006

Kwon, N. H., Fox, P. L., and Kim, S. (2019). Aminoacyl-tRNA synthetases as therapeutic targets. Nat. Rev. Drug Discov. 18, 629-650. doi: 10.1038/s41573019-0026-3

Kwon, N. H., Kang, T., Lee, J. Y., Kim, H. H., Kim, H. R., Hong, J., et al. (2011). Dual role of methionyl-tRNA synthetase in the regulation of translation and tumor suppressor activity of aminoacyl-tRNA synthetase-interacting multifunctional protein-3. Proc. Natl. Acad. Sci. U.S.A. 108, 19635-19640. doi: 10.1073/pnas. 1103922108

Kwon, N. H., Lee, J. Y., Ryu, Y. L., Kim, C., Kong, J., Oh, S., et al. (2018). Stabilization of Cyclin-Dependent Kinase 4 by Methionyl-tRNA Synthetase in p16(INK4a)-Negative Cancer. ACS Pharmacol. Transl. Sci. 1, 21-31. doi: 10.1021/acsptsci.8b00001

Lee, C. W., Chang, K. P., Chen, Y. Y., Liang, Y., Hsueh, C., Yu, J. S., et al. (2015). Overexpressed tryptophanyl-tRNA synthetase, an angiostatic protein, enhances oral cancer cell invasiveness. Oncotarget 6, 21979-21992. doi: 10. 18632/oncotarget. 4273

Lee, J. M., and Kim, T. (2019). Methionyl-tRNA Synthetase is a useful diagnostic marker for lymph node metastasis in non-small cell lung cancer. Yonsei Med. J. 60, 1005-1012. doi: 10.3349/ymj.2019.60.11.1005

Lee, S., Kim, D. G., Kim, K., Kim, T., Lim, S., Kong, H., et al. (2020). 2aminophenylpyrimidines as novel inhibitors of aminoacyl-tRNA synthetase interacting multifunctional protein 2 (AIMP2)-DX2 for lung cancer treatment. J. Med. Chem. 63, 3908-3914. doi: 10.1021/acs.jmedchem.9b0 1765

Li, H., Tian, Y., Li, X., Wang, B., Zhai, D., Bai, Y., et al. (2019). Knockdown of IARS2 inhibited proliferation of acute myeloid leukemia cells by regulating p53/p21/PCNA/eIF4E pathway. Oncol. Res. 27, 673-680. doi: 10.3727/0965 $04018 \times 15426261956343$

Li, Y., Li, X., Cao, M., Jiang, Y., Yan, J., Liu, Z., et al. (2019). Seryl tRNA synthetase cooperates with POT1 to regulate telomere length and cellular senescence. Signal Transd. Target. Therapy 4:50. doi: 10.1038/s41392-019-0078-1

Liang, D., Tian, L., You, R., Halpert, M. M., Konduri, V., Baig, Y. C., et al. (2017). AIMp1 Potentiates T(H)1 Polarization and Is Critical for Effective Antitumor and Antiviral Immunity. Front. Immunol. 8:1801. doi: 10.3389/fimmu.2017. 01801

Liao, X., Hong, Y., Mao, Y., Chen, N., Wang, Q., Wang, Z., et al. (2020). SPH3643: a novel cyclin-dependent kinase 4/6 inhibitor with good anticancer efficacy and strong blood-brain barrier permeability. Cancer Sci. 111, 1761-1773. doi: $10.1111 /$ cas. 14367

Lim, S., Cho, H. Y., Kim, D. G., Roh, Y., Son, S. Y., Mushtaq, A. U., et al. (2020). Targeting the interaction of AIMP2-DX2 with HSP70 suppresses cancer development. Nat. Chem. Biol. 16, 31-41. doi: 10.1038/s41589-019-0415-2

Liu, Q. Q., Li, C. M., Fu, L. N., Wang, H. L., Tan, J., Wang, Y. Q., et al. (2020). Enterotoxigenic Bacteroides fragilis induces the stemness in colorectal cancer via upregulating histone demethylase JMJD2B. Gut Microb. 12:1788900. doi: 10.1080/19490976.2020.1788900

Lu, S., Wang, L. J., Lombardo, K., Kwak, Y., Kim, W. H., and Resnick, M. B. (2020). Expression of Indoleamine 2, 3-dioxygenase 1 (IDO1) and tryptophanyl-tRNA Synthetase (WARS) in gastric cancer molecular subtypes. Appl. Immunohistochem. Mol. Morphol. 28, 360-368. doi: 10.1097/pai. 0000000000000761

Ma, J., Chen, M., Xia, S. K., Shu, W., Guo, Y., Wang, Y. H., et al. (2013). Prostaglandin E2 promotes liver cancer cell growth by the upregulation of FUSE-binding protein 1 expression. Int. J. Oncol. 42, 1093-1104. doi: 10.3892/ ijo.2013.1782

Miyanokoshi, M., Yokosawa, T., and Wakasugi, K. (2018). Tryptophanyl-tRNA synthetase mediates high-affinity tryptophan uptake into human cells. J. Biol. Chem. 293, 8428-8438. doi: 10.1074/jbc.RA117.001247

Mo, Z., Zhang, Q., Liu, Z., Lauer, J., Shi, Y., Sun, L., et al. (2016). Neddylation requires glycyl-tRNA synthetase to protect activated E2. Nat. Struct. Mol. Biol. 23, 730-737. doi: 10.1038/nsmb.3250

Moore, P. A., Jayme, D. W., and Oxender, D. L. (1977). A role for aminoacyl-tRNA synthetases in the regulation of amino acid transport in mammalian cell lines. J. Biol. Chem. 252, 7427-7430.
Mullen, P., Abbott, J. A., Wellman, T., Aktar, M., Fjeld, C., Demeler, B., et al. (2020). Neuropathy-associated histidyl-tRNA synthetase variants attenuate protein synthesis in vitro and disrupt axon outgrowth in developing zebrafish. FEBS J. doi: 10.1111/febs.15449 [Epub ahead of print].

Nam, S. H., Kim, D., Lee, D., Lee, H. M., Song, D. G., Jung, J. W., et al. (2018). Lysyl-tRNA synthetase-expressing colon spheroids induce M2 macrophage polarization to promote metastasis. J. Clin. Invest. 128, 5034-5055. doi: 10.1172/ jci99806

Nam, S. H., Kim, D., Lee, M. S., Lee, D., Kwak, T. K., Kang, M., et al. (2015). Noncanonical roles of membranous lysyl-tRNA synthetase in transducing cellsubstrate signaling for invasive dissemination of colon cancer spheroids in $3 \mathrm{D}$ collagen I gels. Oncotarget 6, 21655-21674. doi: 10.18632/oncotarget.4130

Oh, A. Y., Jung, Y. S., Kim, J., Lee, J. H., Cho, J. H., Chun, H. Y., et al. (2016). Inhibiting DX2-p14/ARF interaction exerts antitumor effects in lung cancer and delays tumor progression. Cancer Res. 76, 4791-4804. doi: 10.1158/0008-5472. can-15-1025

Pacher, M., Seewald, M. J., Mikula, M., Oehler, S., Mogg, M., Vinatzer, U., et al. (2007). Impact of constitutive IGF1/IGF2 stimulation on the transcriptional program of human breast cancer cells. Carcinogenesis 28, 49-59. doi: 10.1093/ carcin/bgl091

Paley, E. L., Paley, D. E., Merkulova-Rainon, T., and Subbarayan, P. R. (2011). Hypoxia signature of splice forms of tryptophanyl-tRNA synthetase marks pancreatic cancer cells with distinct metastatic abilities. Pancreas 40, 1043-1056. doi: 10.1097/MPA.0b013e318222e635

Pang, Y. L., Poruri, K., and Martinis, S. A. (2014). tRNA synthetase: tRNA aminoacylation and beyond. Wiley Interdiscip. Rev. RNA 5, 461-480. doi: 10. 1002/wrna. 1224

Park, B. J., Kang, J. W., Lee, S. W., Choi, S. J., Shin, Y. K., Ahn, Y. H., et al. (2005). The haploinsufficient tumor suppressor p18 upregulates p53 via interactions with ATM/ATR. Cell 120, 209-221. doi: 10.1016/j.cell.2004.11.054

Park, M. C., Kang, T., Jin, D., Han, J. M., Kim, S. B., Park, Y. J., et al. (2012). Secreted human glycyl-tRNA synthetase implicated in defense against ERKactivated tumorigenesis. Proc. Natl. Acad. Sci. U.S.A. 109, E640-E647. doi: 10.1073/pnas.1200194109

Park, S. G., Schimmel, P., and Kim, S. (2008). Aminoacyl tRNA synthetases and their connections to disease. Proc. Natl. Acad. Sci. U.S.A. 105, 11043-11049. doi: 10.1073/pnas.0802862105

Pierce, C. J., and Simmons, J. L. (2020). BRN2 expression increases anoikis resistance in melanoma. Oncogenesis 9:64. doi: 10.1038/s41389-020-00247-1

Qi, L., Zhou, B., Chen, J., Hu, W., Bai, R., Ye, C., et al. (2019). Significant prognostic values of differentially expressed-aberrantly methylated hub genes in breast cancer. J. Cancer 10, 6618-6634. doi: 10.7150/jca.33433

Qin, X., Li, C., Guo, T., Chen, J., Wang, H. T., Wang, Y. T., et al. (2017). Upregulation of DARS2 by HBV promotes hepatocarcinogenesis through the miR-30e-5p/MAPK/NFAT5 pathway. J. Exp. Clin. Cancer Res. 36:148. doi: 10. 1186/s13046-017-0618-x

Salzer, W., Bostrom, B., and Messinger, Y. (2018). Asparaginase activity levels and monitoring in patients with acute lymphoblastic leukemia. Leukem. Lymphoma 59, 1797-1806. doi: 10.1080/10428194.2017.1386305

Sankaranarayanan, R., Dock-Bregeon, A. C., Rees, B., Bovee, M., Caillet, J., Romby, P., et al. (2000). Zinc ion mediated amino acid discrimination by threonyl-tRNA synthetase. Nat. Struct. Biol. 7, 461-465. doi: 10.1038/75856

Shafabakhsh, R., Yousefi, B., Asemi, Z., Nikfar, B., Mansournia, M. A., and Hallajzadeh, J. (2020). Chitosan: a compound for drug delivery system in gastric cancer-a review. Carbohydr. Polym. 242:116403. doi: 10.1016/j.carbpol.2020. 116403

Shin, S. H., Kim, H. S., Jung, S. H., Xu, H. D., Jeong, Y. B., and Chung, Y. J. (2008). Implication of leucyl-tRNA synthetase 1 (LARS1) over-expression in growth and migration of lung cancer cells detected by siRNA targeted knock-down analysis. Exp. Mol. Med. 40, 229-236. doi: 10.3858/emm.2008.40.2.229

Sivaraman, A., Kim, D. G., Bhattarai, D., Kim, M., Lee, H. Y., Lim, S., et al. (2020) Synthesis and structure-activity relationships of arylsulfonamides as AIMP2DX2 inhibitors for the development of a novel anticancer therapy. J. Med. Chem. 63, 5139-5158. doi: 10.1021/acs.jmedchem.9b01961

Stephen, J., Nampoothiri, S., Banerjee, A., Tolman, N. J., Penninger, J. M., Elling, U., et al. (2018). Loss of function mutations in VARS encoding cytoplasmic valyl-tRNA synthetase cause microcephaly, seizures, and progressive cerebral atrophy. Hum. Genet. 137, 293-303. doi: 10.1007/s00439-018-1882-3 
Suh, J. H., Park, M. C., Goughnour, P. C., Min, B. S., Kim, S. B., Lee, W. Y., et al. (2020). Plasma Lysyl-tRNA synthetase 1 (KARS1) as a novel diagnostic and monitoring biomarker for colorectal cancer. J. Clin. Med. 9:533. doi: 10.3390/ jcm9020533

Tang, X., Ding, C. K., Wu, J., Sjol, J., Wardell, S., Spasojevic, I., et al. (2017). Cystine addiction of triple-negative breast cancer associated with EMT augmented death signaling. Oncogene 36, 4235-4242. doi: 10.1038/onc.2016.394

Tian, T., Xiao, L., Du, J., Zhu, X., Gu, Y., Qin, N., et al. (2017). Polymorphisms in CARS are associated with gastric cancer risk: a two-stage case-control study in the Chinese population. Gastr. Cancer 20, 940-947. doi: 10.1007/s10120-0170717-6

Wang, D., Zhao, R., Qu, Y. Y., Mei, X. Y., Zhang, X., Zhou, Q., et al. (2018). Colonic Lysine homocysteinylation induced by high-fat diet suppresses DNA damage repair. Cell Rep. 25, 398-412.e396. doi: 10.1016/j.celrep.2018.09.022

Wellman, T. L., Eckenstein, M., Wong, C., Rincon, M., Ashikaga, T., Mount, S. L., et al. (2014). Threonyl-tRNA synthetase overexpression correlates with angiogenic markers and progression of human ovarian cancer. BMC Cancer 14:620. doi: 10.1186/1471-2407-14-620

Wu, J., Subbaiah, K. C. V., Xie, L. H., Jiang, F., Khor, E. S., Mickelsen, D., et al. (2020). Glutamyl-Prolyl-tRNA synthetase regulates proline-rich pro-fibrotic protein synthesis during cardiac fibrosis. Circ. Res. 127, 827-846. doi: 10.1161/ circresaha.119.315999

Yang, P. P., Yu, X. H., and Zhou, J. (2020). Tryptophanyl-tRNA synthetase (WARS) expression in uveal melanoma - possible contributor during uveal melanoma progression. Biosci. Biotechnol. Biochem. 84, 471-480. doi: 10.1080/09168451. 2019.1686967

Yeom, E., Kwon, D. W., Lee, J., Kim, S. H., Lee, J. H., Min, K. J., et al. (2020). Asparaginyl-tRNA synthetase, a novel component of hippo signaling, binds to salvador and enhances yorkie-mediated tumorigenesis. Front. Cell Dev. Biol. 8:32. doi: $10.3389 /$ fcell.2020.00032

Yin, J., Liu, W., Li, R., Liu, J., Zhang, Y., Tang, W., et al. (2016). IARS2 silencing induces non-small cell lung cancer cells proliferation inhibition, cell cycle arrest and promotes cell apoptosis. Neoplasma 63, 64-71. doi: 10.4149/neo_2016_008

Yum, M. K., Kang, J. S., Lee, A. E., Jo, Y. W., Seo, J. Y., Kim, H. A., et al. (2016). AIMP2 controls intestinal stem cell compartments and tumorigenesis by modulating Wnt/ $\beta$-Catenin signaling. Cancer Res. 76, 4559-4568. doi: 10. 1158/0008-5472.can-15-3357

Zhang, C., Lin, X., Zhao, Q., Wang, Y., Jiang, F., Ji, C., et al. (2020). YARS as an oncogenic protein that promotes gastric cancer progression through activating
PI3K-Akt signaling. J. Cancer Res. Clin. Oncol. 146, 329-342. doi: 10.1007/ s00432-019-03115-7

Zhang, J., Niu, D., Sui, J., Ching, C. B., and Chen, W. N. (2009). Protein profile in hepatitis B virus replicating rat primary hepatocytes and HepG2 cells by iTRAQ-coupled 2-D LC-MS/MS analysis: insights on liver angiogenesis. Proteomics 9, 2836-2845. doi: 10.1002/pmic.200800911

Zhong, L., Zhang, Y., Yang, J. Y., Xiong, L. F., Shen, T., Sa, Y. L., et al. (2015). Expression of IARS2 gene in colon cancer and effect of its knockdown on biological behavior of RKO cells. Int. J. Clin. Exp. Pathol. 8, 1215112159.

Zhong, Q., Liu, Z. H., Lin, Z. R., Hu, Z. D., Yuan, L., Liu, Y. M., et al. (2018). The RARS-MAD1L1 fusion gene induces cancer stem cell-like properties and therapeutic resistance in nasopharyngeal carcinoma. Clin. Cancer Res. 24, 659-673. doi: 10.1158/1078-0432.ccr-17-0352

Zhou, X., Han, S., Wilder-Romans, K., Sun, G. Y., Zhu, H., Liu, X., et al. (2020). Neddylation inactivation represses androgen receptor transcription and inhibits growth, survival and invasion of prostate cancer cells. Neoplasia 22, 192-202. doi: 10.1016/j.neo.2020.02.002

Zhou, Z., Sun, B., Huang, S., and Yu, D. (2020). Roles of aminoacyl-tRNA synthetase-interacting multi-functional proteins in physiology and cancer. Cell Death Dis. 11:579. doi: 10.1038/s41419-020-02794-2

Zhou, X., Xue, L., Hao, L., Liu, S., Zhou, F., Xiong, H., et al. (2013). Proteomicsbased identification of tumor relevant proteins in lung adenocarcinoma. Biomed. Pharmacother. 67, 621-627. doi: 10.1016/j.biopha.2013. 06.005

Zhu, C., Sun, B., Nie, A., and Zhou, Z. (2020). The tRNA-associated dysregulation in immune responses and immune diseases. Acta Physiol. 228:e13391. doi: 10.1111/apha.13391

Conflict of Interest: The authors declare that the research was conducted in the absence of any commercial or financial relationships that could be construed as a potential conflict of interest.

Copyright (c) 2020 Zhou, Sun, Nie, Yu and Bian. This is an open-access article distributed under the terms of the Creative Commons Attribution License (CC BY). The use, distribution or reproduction in other forums is permitted, provided the original author(s) and the copyright owner(s) are credited and that the original publication in this journal is cited, in accordance with accepted academic practice. No use, distribution or reproduction is permitted which does not comply with these terms. 